\title{
The Effect of Intraspinal Micro Stimulation With Variable Stimulating Pattern in Adult Rat With Induction of Spinal Cord Injury in the Treatment of Spinal Cord Injuries
}

\author{
Mohaddeseh Hedayatzadeh $^{1 \oplus}$, Hamid Reza Kobravi $^{1 *(\infty)}$, Maryam Tehranipour $^{2}$ \\ ${ }^{1}$ Department of Medical Engineering, Faculty of Engineering, Islamic Azad University, Mashhad Branch, Iran \\ ${ }^{2}$ Department of Biology, Faculty of Science, Islamic Azad University, Mashhad Branch, Iran
}

\begin{abstract}
Background: Spinal cord injury is one of the diseases that, no specific treatment has yet found despite the variety of works that have done in this field. Different approaches to treat such injuries have investigated today. One of them is invasive intra-spinal interventions such as electrical stimulation. Therefore, in this study, the effect of the protocol for intra-spinal variable and fixed electrical stimulation has been investigated in order to recover from spinal cord injury.

Methods: In the study, 18 Wistar male rats randomly divided into Three groups, including intraspinal electrical stimulation (IES), IES with variable pattern of stimulation (VP IES) and a sham group. Animals initially subjected to induced spinal cord injury. After one week, the animal movement was recorded on the treadmill during practice using a camera and angles of the ankle joint were measured using the Tracker software. Then, the obtained data were analyzed by nonlinear evaluations in the phase space.

Results: The motion analyses and kinematic analyses were carried out on all groups. According to the achieved results, the gait dynamics of the VP IES group has the most conformity to the gait dynamics of the healthy group. Also, the best quality of the balance preservation observed in the VP IES group.

Conclusion: It can be concluded that the IES with variable pattern of stimulation along with exercise therapy has significant gait restorative effects and increases the range of motion in rats with induced spinal cord injury.

Keywords: Spinal cord injury; Motion recovery; Intraspinal microstimulation; Rat; Phase space; Geometric feature; Synergy pattern.
\end{abstract}

\author{
*Correspondence to \\ Hamid Reza Kobravi, \\ Research Center of Biomedical \\ Engineering, Islamic Azad \\ University, Mashhad Branch, \\ Mashhad, Iran. \\ Tel: +985136629467; \\ Email: hkobravi@mshdiau.ac.ir
}

Published online August 31, 2019

Citation: Hedayatzadeh M, Kobravi HR, Tehranipour M. The Effect of intraspinal micro stimulation with variable stimulating pattern in adult rat with induction of spinal cord injury in the treatment of spinal cord injuries. Int Clin Neurosci J. 2019;6(3):83-91. doi:10.15171/ icnj.2019.17.

\section{Introduction}

Spinal cord injury may occur due to an accident, illness. ${ }^{1,2}$ Such injuries may cause degeneration of the spinal cord completely or partially. ${ }^{1,2}$ When the spinal cord is damaged, the connection between the spinal cord and the brain is affected, and messages are not sent correctly from the brain to the spinal cord to carry out activities.

For this reason, people with spinal injury suffer from sensory and motor disorders after the damage. ${ }^{3}$ The inability to reproduce damaged nerve cells in the central nervous system is a factor that has led to a lack of finding precise treatment for spinal cord injury. ${ }^{4,5}$ Motor rehabilitation is a major scientific challenge for researchers in spinal cord injuries despite scientific advances in the fields of neuroscience, neurological rehabilitation. ${ }^{6}$

Therefore, motor rehabilitation in people with paralyzed spinal cord injury is one of the most respected research areas in the field of neurological rehabilitation.

However, in recent years, many efforts have been made in the use of stem cells, reproduction of neural cells. However, the only effective way to help these patients at present is through the use of up-to-date motor rehabilitation. ${ }^{7-9}$ Meanwhile, electrical stimulation of the spinal cord considered as a potentially effective strategy. Motor commands in such a strategy applied to the motor nerves as internal electrical stimulation signals. The rate of using motor units of muscles as well as the number of stimulated motor units of muscles can control by adjusting the intensity of the stimulation signals over time. In this way, the strength of the contraction of the muscle and thus, the formation of the torque around the joints will control. The application of electrical stimulation with 
appropriate and simultaneous stimulation patterns in the muscles involved in motion can cause rehabilitation of a motion. ${ }^{8-10}$ According to several decades of investigation by researchers in this field, acceptable performance of this strategy has shown in the rehabilitation of many motor functions. However, the use of intra-spinal electric stimulation in creating and controlling complex movements such as gait face serious challenges. ${ }^{11-13}$ Therefore, researchers in this area seek more effective solutions in order for gait control. For ages, researchers have indicated that there are neural circuits in the spinal cord, which generate neural orders related to the control of lower rhythmic motion. ${ }^{14-16}$ The fascinating point is that the mentioned neural circuits can produce neural commands for rhythmic movement even without the effects of afferents and without receiving neural signals from the brain. Such neural circuits are called central pattern generators. ${ }^{17-19}$

Researchers have shown with abundant empirical evidence in animals and humans that deep electrical stimulation of the spinal cord can restore the activity of the central nervous system. As a result of this process, gait can be retrieved. ${ }^{8,20-22}$ In all mentioned works, stimulation signals delivered to the spinal epidural region. The delivered electrical signals are periodic pulses. The intensity of then can adjust by turning the pulse width, pulse amplitude, and pulse frequency. In each reported researchers, the parameters of the stimulation pulses were adjusted once according to the situation of the animal. In other words, the determined stimulation pattern fixed in all sessions of motor exercises. In other words, though using the periodic stimulation pulses is inevitable, but there is no typical stimulation profile for intraspinal stimulation. Also, none of the mention works had evaluated the effect of electrical stimulation with a variable pattern of stimulation. The variable pattern of stimulation means designing a rehabilitation intervention with varying intensity. Thus, this study aimed to evaluate and to compare the effect of rehabilitation intervention with fixed intensity and with variable intensity. The goal in the current article is to obtain the best pattern of electrical stimulation of the spinal cord and the proper location of these stimuli to maximize motor rehabilitation. Also, so far, no analysis has been done on the dynamics of nonlinear motion. Hence, in the present study, nonlinear analyzes on animal kinematic data were performed due to motor exercises with intra-spinal electrical stimulation and changes which have made in the dynamics of animal movement by the mentioned stimuli have been investigated.

\section{Material and Methods \\ Animal and Housing Conditions \\ Eighteen male, Wistar rats, weighing between 200-250 $\mathrm{g}$ served as subjects for these experiments. All rats were group-housed in standard Plexiglas cages (3 to 4 rats per}

cage) with free access to food and water and 12-12 lightdark cycle, with lights on at $6.00 \mathrm{~h}$. Ambient temperature in the animal facility kept at $22 \pm 2 \mathrm{C}^{\circ}$. All procedures were under the local guidelines for the care and use of laboratory animals and approved by the Islamic Azad University (Mashhad branch).

\section{Groups}

The experimental animals randomly divided into 4 groups, and the number of animals in each group were $\operatorname{six}(n=6)$.

- Intra-spinal electrical stimulation group (IES)

- Intra-spinal electrical stimulation group with variable stimulating pattern (VP IES)

- The control group (Sham)

For control group, only spinal cord injury was induced.

Induction of Spinal Cord Injury in Animals and Implantation of the Stimulation Electrode

At first, animals anesthetized with an intraperitoneal injection of ketamine $(100 \mathrm{mg} / \mathrm{kg})$ and xylazine $(20 \mathrm{mg} /$ $\mathrm{kg}$ ). Antibiotic (intramuscular with a dose of $50 \mathrm{ml} /$ $\mathrm{kg}$ ) and morphine (intraperitoneal with $5 \mathrm{~mL} / \mathrm{kg}$ ) were also used to reduce the pain caused by the surgery. The body temperature maintained at $37^{\circ} \mathrm{C}$, and all surgical procedures performed under sterile conditions.

When the appropriate level of anesthesia achieved, the hair on the back of the animal shaved for surgery. Then, the surface disinfected and a longitudinal incision was made at the back of the skin in the region equivalent to T10 in the spinal cord to induce spinal cord injury. Fat and muscle removed at the level of the mentioned vertebrae in the segment T10 of animal, spinal cord lesion-induced. The ground electrode was attached to the muscles and the stimulating electrode placed on the segment T11 of the spinal cord. In order to plant a stimulating electrode after laminectomy in the segment T11, a hole made by 20-gauge injection needle and spring-shaped tube, (made of stainless steel) placed within it. The stimulating electrode inserted into the tube and liquid glue used the site of their junction. Before guiding the electrode into the tube, it has tied to the upper vertebra to fixed entirely. The ground electrode was tied to the lower spine to ensure lack of displacement since the mentioned electrode displaced in the previous methods. Several methods were applied for planting and fixing the electrode at the desired location since the main problem in the study was the inability to fix the electrode at the desired point, which was ultimately achieved by the method mentioned above. In general, one of the most deciding issues in this study is choosing the best method for spinal cord injury with the least possible damage in the surrounding tissues and skeletal structure of the animal with the induction of the least inflammatory effects.

Therefore, after induction of spinal cord injury, the 
stimulating electrode was installed at the nearest site to the location of the lesion in a lower segment so that the least damage exerted to the animal. This method reduces the risk of infection at the site of the lesion. After the successful induction of spinal cord injury, finding the best method and pattern of intra-spinal electrical stimulation is considered. As it previously stated, the mouse suffered a spinal cord injury after the anesthesia in the segment T10 and then stimulating electrode was attached to the segment T11 and reference electrode connected to the muscles, near the planting site of stimulating electrode. Afterward, the electrodes fixed in that location. After a period of restoration and recovery (administration of antibiotics and appropriate analgesics), electrical stimulation of the spinal cord with the same patterns (The same and variable frequency, amplitude, and pulse) was applied to obtain the best motor result in intra-spinal electrical stimulation groups with constant and variable stimulation patterns.

\section{Postoperative Care}

Ethical guidelines for working with laboratory animals were considered to minimize the probable suffering of animals. In order to begin motion training and stimulation, animals individually kept with water and food after surgery, and their health status continuously evaluated. The bladder was also examined daily and discharged. Draining of the rat bladder was needed twice a day after the sci to return the bladder reflex. This procedure is done by touching the abdominal area of the animal to determine whether the bladder is full or not. In case of being full, it slightly drained by the pressure exertion. Every day over 3 days, antibiotics $(50 \mathrm{~mL} / \mathrm{kg}$ of enrofloxacin) in the form of intramuscular injection was used to prevent infections caused by induction of lesion in the spine.

Moreover, morphine (5 $\mathrm{mL} / \mathrm{kg})$ was injected intraperitoneally to reduce pain and The lactate ringer solution $(5 \mathrm{cc}$ ) was taken subcutaneously in order to maintain hydration. Postoperative care has been taken to prevent animal death. Among other reasons of mortality rate, it can refer to inappropriate material of the electrodes and an increase in the dose of anesthetic drug (in some cases, due to the lack of anesthesia of the animal because of injecting the drug into an inappropriate site); therefore, such points should take into account. Standard methods related to the International Association for the Study of Pain (IASP) have used at all stages of this research, including surgery, implantation of stimulating electrodes, and motor exercises.

\section{Stimulation Protocol}

Intra-spinal micro-stimulation (ISMS) is an approach of functional electrical stimulation targeting the lumbosacral circuit. A silver stimulating electrode (silver, A-M Systems, USA) placed at a depth of $1.5 \mathrm{~mm}$ from the spinal cord.
Stimulation of this area causes the coordinated movement of the leg. A characteristic feature of this method is the gradual application of force due to its order and the use of fatigue-resistant motor units.

This feature has attributed to activation of the synaptic transmission of the motor neurons through a vast network of projection of sensory afferents and interneurons that have been activated by spinal stimulation.

In sum, this approach is a practical way to restore the capacity of standing and gait after spinal cord injury. Lumbosacral circuit of mammalians (rat, rabbit, and cat) with spinal cord injuries can use sensory information by epidural stimulation to produce a wide range of movements, including stepping and standing.

It has anticipated that motor training with epidural stimulation improves the capacity of the spinal cord to produce motion. As previously mentioned, the stimuli in this study applied in the form of two stimulating groups. Intra-spinal electrical stimulation group with a constant stimulation pattern which applied with frequency characteristics of $40 \mathrm{~Hz}$ and amplitude of 3 as well as a pulse duration of 200 microseconds for 15 minutes per period. The intra-spinal electrical stimulation group with a variable stimulation pattern which applied with varying frequency characteristics between $40-60 \mathrm{~Hz}$, amplitude between 3-16 V, and a pulse duration of 200 microseconds for 15 minutes per period.

The frequency, intensity, and location of the stimulus are significant parameters like the generated movements, among which the most effective frequency is between 40 and $60 \mathrm{~Hz}$. Stimulation at lower frequencies $(40 \mathrm{~Hz})$ adjusts the rhythmic pattern between the limb flexors and extensors poorly while higher frequencies $(60 \mathrm{~Hz})$ causes irregular patterns of movement. For this purpose, the frequency pattern has considered between the indicated values in the stimulating group with the variable. In the stimulation group with the variable pattern, the intensity of electrical stimulation during the sessions has increased. In this way, the effect of increasing the intensity of intervention during intervention and motor exercise has evaluated. So that the amplitude and frequency of the stimulation in each session of the training has increased significantly compared to the previous session in a way that the difference in the motor's response of the animal to the previous session is considerable. In general, it can express that tonic stimulation of the dorsal surface of the lumbosacral spinal cord through the electrode placed on the Dura plane can induce some movements in rats with defective or complete spinal cord injury, cats and humans.

The frequency and phase of the rhythmic neurological instructions of the spinal cord can control by adjusting the intensity of stimulation and the use of the variable stimulating protocol. In this way, not only can the recovery of gait be retrieved, but also the quality of motion can be controlled using the variable stimulating protocol. 


\section{The Protocol of Motor Exercises}

For more than a decade, studies related to gait practice on the treadmill with body weight support on animals have indicated an improvement in walking performance after spinal cord injury.

In the present study, the rat was placed on a treadmill by a suspension system so that both the hands and feet of the rat touched the treadmill surface in order to practice walking on the treadmill. During the exercise session, electrical stimulation signals applied to the epidural region with $40 \mathrm{~Hz}$ frequency characteristics, $3 \mathrm{~V}$ amplitude and pulse duration 200 microseconds for 15 minutes in a constant stimulation group as well as varying frequency characteristics between 40-60 Hz, variable amplitude between the range of 3-16 volts and pulse duration of 200 microseconds for 15 minutes in the stimulation group with variable stimulation pattern. Daily exercise sessions were performed at two times in the morning and afternoon with a 12-hour interval for 14 days. In order to measure the angles of the joint, color markers placed on each joint. Two digital cameras were used to record the angle of the joints during stimulation. Cameras were installed perpendicularly to the plane where the right and left feet of the rat located. In the present study, the aim of measuring the position of angles of the right and left ankles was investigating nonlinear analyzes on the phase plane to measure the amount of motor recovery. Images were imported to the computer instantly at 60 frames per second, and the position of the markers and the joints have extracted.

\section{Evaluation Based on Nonlinear Analysis}

After recording the position and coordinates of the angle of the ankle during training, the coordinates for each foot were separately mapped to phase space separately, and the proper features in this space examined. Occurred changes in the system dynamics, the pattern of muscle coordination, and the method of their interaction can observe on the phase plane. Therefore, in the current research, analysis of Changes in whole system behavior and behavioral similarities in position changes of ankle joint in the rotating plane has been investigated between the healthy animals and those under treatment, and thus changes in the state variables are evaluated in relation to each other.

In general, a set of points, which recorded in succession at a given time, such as recorded situations from angles of animal ankle joint is called time series. The time series analysis focuses on the fact that these points have a significant structure. The path of identifying the unknown characteristics of the system based on these observations to understand the dynamics of the system is called dynamic analysis of time series. Dynamics of the system should be taken to the state space so that relativity of the dynamic properties of the system can extract from its time series.

The most important technique for phase reconstruction is the use of the time delay method. In this way, the vectors represented in a new space by using the delayed values of the measurements. If the time series $x(n)$ for $n=1,2,3$, ......, $\mathrm{N}$ is given, time delay vectors of phase space formed as follows.

$$
\begin{aligned}
& X(i)=[x(i), x(i+\tau), x(I, 2 \tau), \ldots, x(I+(m-1) \tau)] \\
& i=1,2, \ldots, N-(m-1) \tau
\end{aligned}
$$

Where $\tau$ is the time delay and $m$ is the dimension of space. Thus, the system state at any time is shown by the vector $\mathrm{x}(\mathrm{i})$ in the phase space. Choosing different values of $m$ and $\tau$ will result in different reconstructed state vectors. In the present research, after transferring the position of the ankle joint to the phase space, dynamic and qualitative characteristics of the signal can be achieved by calculating the geometric properties that have a low rate of computational volume. These features also provide information on how the position of changes in the ankle joint angle, and their error is less than linear characteristics. In other words, these attributes adopted as indicators that contain information about the synergy pattern.

\section{Feature Extraction}

Phase space is one of the essential tools used to understand the behavior and dynamics of nonlinear systems. Behavioral characteristics can be expressed appropriately by using this tool in a qualitative way feature Extraction is a useful method for mining information. In the present research, five features related to the phase plane of the pattern of changes in the position of the angle of the ankle joint have investigated. Three features are geometric and qualitative, and two other ones are dynamic and qualitative in phase space. The desired features are Central Gravity, Correlation Sum.

\section{Central Gravity}

Center of gravity is one of the geometric characteristics as a descriptor of a shape. It is also called center of mass. The position of the center of mass relative to the shape is constant. If $\left(\mathrm{x}_{\mathrm{i}}, \mathrm{Yi}_{\mathrm{i}}\right)$ are the positions of the points in the phase plane, the geometric indices of the center of mass can calculate as the following equation. If the equation of the implanted region clearly characterized, the center of mass $\left(g_{x}, g_{y}\right)$ can define as the following equations.

$\left\{\begin{array}{l}g_{x}=\frac{1}{N} \sum_{i=1}^{N} x_{i} \\ g_{y}=\frac{1}{N} \sum_{i=1}^{N} y_{i}\end{array}\right.$

In the equation mentioned above, $N$ represents the number of points $\left(\mathrm{x}_{\mathrm{i}}, \mathrm{y}_{\mathrm{i}}\right)$ in the figure. If the equation of 
shape contour is specified, the center of mass $\left(\mathrm{g}_{\mathrm{x}}, \mathrm{g}_{\mathrm{y}}\right)$ can state as the following equations.

$\left\{\begin{array}{l}g_{x}=\frac{1}{6 A} \sum_{i=0}^{N-1}\left(x_{i}+x_{i+1}\right)\left(x_{i} y_{i+1}-x_{i+1} y_{i}\right) \\ g_{y}=\frac{1}{6 A} \sum_{i=0}^{N-1}\left(y_{i}+y_{i+1}\right)\left(x_{i} y_{i+1}-x_{i+1} y_{i}\right)\end{array}\right.$

Where $\mathrm{A}$ is the area of the contour, and it expressed as below:

$A=\frac{1}{2}\left|\sum_{i=0}^{N-1}\left(x_{i} y_{i+1}-x_{i+1} y_{i}\right)\right|$

In this method, the location of the center of mass does not depend on the distribution of shape points.

\section{Correlation Sum}

The sum of correlations is a correlation integral estimator, which represents the probability average.

$$
C(R)=\frac{1}{N(N-1)} \sum_{i=1}^{N} \sum_{j=1}^{N} \Theta\left(R-\left|x_{i}-x_{j}\right|\right)
$$

Where $\mathrm{R}$ is the Euclidean distance between the two points of $x_{i}$ and $x_{i}, C(R)$ is the sum of the correlations, $\mathrm{N}$ is the total number of curve path points, and $\Theta$ is the Heaviside step function which defined as the following relation:

$$
\begin{array}{lll}
\Theta(x)=0 & \text { if } & x \prec 0 \\
\Theta(x)=0 & \text { if } & x \geq 0
\end{array}
$$

According to the mentioned relation, for every $\mathrm{x}_{\mathrm{j}}$ that situated at a distance $\mathrm{R}$ from the point $\mathrm{x}_{\mathrm{i}}$, the value of the Heaviside step function is equal to 1 ; otherwise, its value is zero.

\section{Assessment of Motor Behavior Motor Coordination}

A test that was used to investigate motor behavior is the Rotarod rat. It is used to investigate motor ability in the rat, which evaluates various parameters such as running time and endurance, balance, and motor coordination. During the test, the animal positioned on a rotating Rod. The height of the device is designed not to be too high so that the animal does not suffer from damage due to

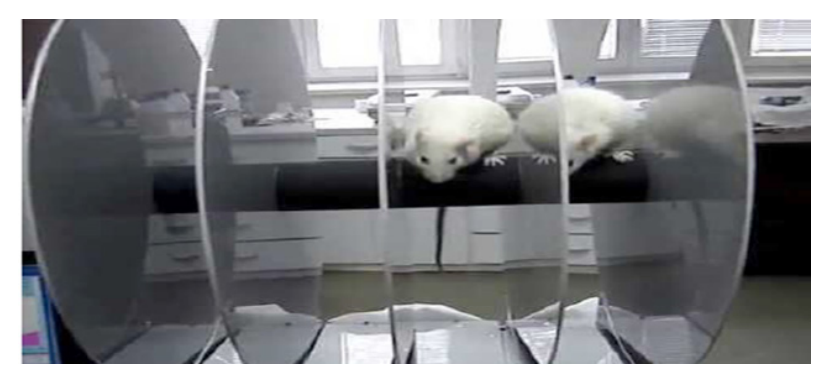

Figure 1. Test Recording Using the Rotarod Rat. its falling. It is also not short enough to cause the state of consciousness of the animal induced in case of falling. A rodent instinctively tries to stay on the cylinder and not to fall. The amount of time the animal remains on the rotating Rod considered as a marker of balance, muscular coordination, and physical conditions of the animal.

Table 1 shows the technical characteristics of the device (manufactured by Borj Sanat Azma Co).

\section{Results}

\section{Results of Nonlinear Analyses}

In the current paper, indicators, that have had the least difference and the most similarity among the various groups, have been extracted in the phase space by processing the position of the angle of the joints recorded from the ankles of the animals during exercise.

The phase space was plotted for the right and left ankle joint angles in a motor cycle for all three groups. The plotted phase spaces are related to the last exercise session in groups.

Finally, the motor recovery rate investigated due to the pattern created on the phase plane of each group, relative to the pattern of the healthy animal phase plane. Then, simple geometric features with less computational errors and volume obtained for each cycle in the phase plane. Figure 2 shows the pattern of changes in the right and left ankle joint angles of the animals. Table 2 describes the analyzed geometric features extracted from the phase space. Tables 3 indicates the mean of the geometric features related of the right foot in all groups, and Table 4 shows the mean of the extracted geometric features related to the left foot. As it can see, among the results obtained of healthy animal and group (VP IES), all of the gained characteristics have remarkable similarities, and indeed, they have the least deviations than their average. In other words, the geometric properties examined in these two groups are most similar to each other. The similarities, related to the changes in the position of the joint and the pattern of concurrent activity, indicate a similarity of motor behavior between the two groups. Since the outcomes achieved in the electrical stimulation group with a variable stimulating pattern has the most similarity in terms of geometric characteristics to healthy animals, there was more and better motor coordination recovery which can mean improvement of the ability of the musculoskeletal system in animal to maintain balance.

Table 1. The Technical Characteristics of the Device

\begin{tabular}{ll}
\hline Dimensions & $45(\mathrm{w}) \times 49(\mathrm{~d}) \times 53(\mathrm{~h}) \mathrm{cm}$ \\
Revolving Speed & $5-45 \mathrm{rpm}$ \\
Display & $\begin{array}{l}\text { Date, time, revolving speed, cut-off time, lapse } \\
\text { time of each channel (3 digits) }\end{array}$ \\
Weight & $15.50 \mathrm{~kg}$ \\
Power Requirements & $220 \mathrm{~V} \mathrm{50/60} \mathrm{Hz}$ \\
\hline
\end{tabular}




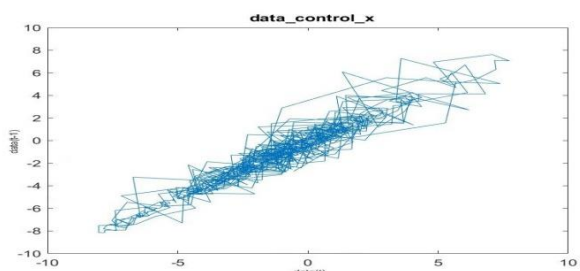

A

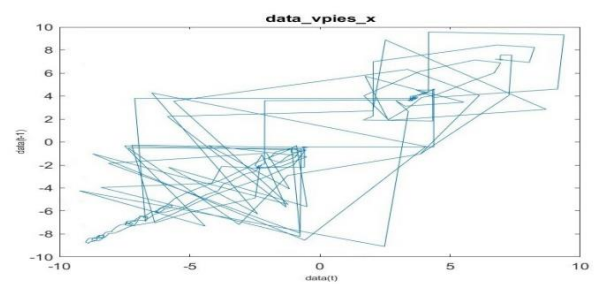

C

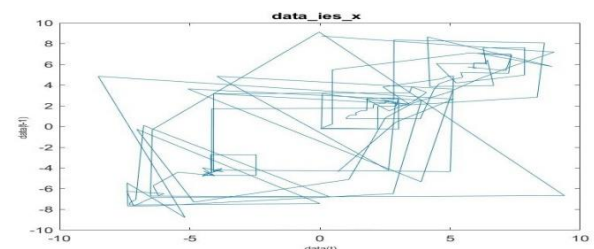

E

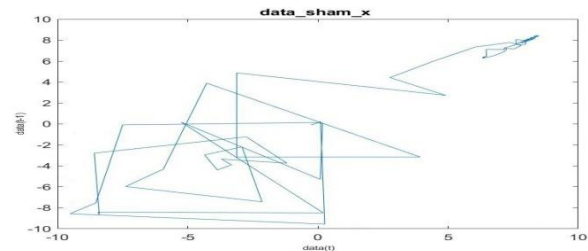

G

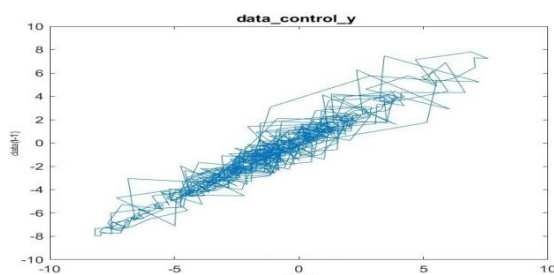

B

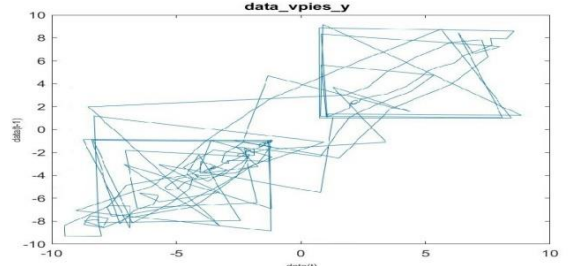

D

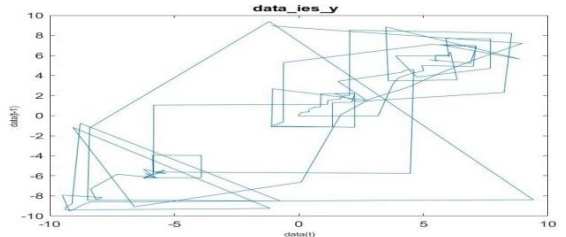

F

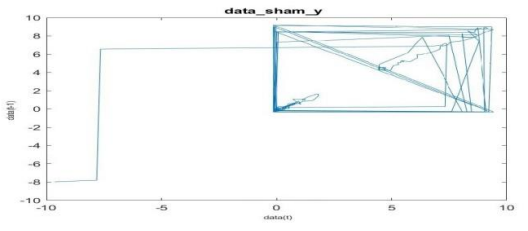

H

Figure 2. A: phase space of the angle of healthy animal's left ankle. B: phase space of the angle of healthy animal's right ankle. C: Phase space of the angle of the left ankle in the electrical stimulation group with the variable stimulating protocol. D: Phase space of the angle of the right ankle in the electrical stimulation group with the variable stimulating protocol. E: Phase space of the angle of the left ankle in the electrical stimulation group with the constant stimulating protocol. F: Phase space of the angle of the right ankle in the electrical stimulation group with the constant stimulating protocol. G: Phase space obtained from the angle of the left ankle of the sham group. $\mathrm{H}$ : Phase space obtained from the angle of right ankle of the sham group.

\section{Motor Coordination}

In Figure 3, the test results indicate the time it takes for the animal to remain on the Rotating Rod, which considered as a marker of balance, muscular coordination, and physical conditions of the animal. As it has shown in Figure 3, the group (VP IES) has been able to stay longer

Table 2. Geometric Features of Phase Space

\begin{tabular}{lll}
\hline Feature & Symbol & Description \\
\hline 1 & $\mathrm{Gx}$ & The horizontal center of gravity \\
2 & $\mathrm{G}_{\mathrm{y}}$ & The vertical center of gravity \\
3 & $\mathrm{C}_{\mathrm{R}}$ & Correlation sum \\
\hline
\end{tabular}

on the Rotating Rod. This suggests that the animal has been able to make better muscular coordination to balance and stay on the rotating Rod. As a result, motor recovery in this group has been better than other groups.

\section{Discussion}

Finding an effective treatment for spinal cord injuries has always been one of the goals of the study and research of scientists and researchers in this field. In the current study, the effect of electrical stimulation with variable and constant stimulating protocols in animals by induction of spinal cord injury investigated for motor recovery. In fact, according to the present study on animals in which their

Table 3. Average Accuracy of Geometric Features for the Right Angle of the Animal Groups

\begin{tabular}{lcccc}
\hline Feature & ISE Group & VP ISE Group & Intact Animal Group & Sham Group \\
\hline Gx & 1.5174 & 1.4968 & 1.1662 & 4.4068 \\
Gy & 1.5037 & 1.4879 & 1.1374 & 4.4396 \\
CR & 1.7280 & 1.7329 & 2.5826 & 0.6338 \\
\hline
\end{tabular}


Table 4. Average Accuracy of Geometric Features for the Left Angle of the Animal Groups

\begin{tabular}{lcccc}
\hline Feature & ISE Group & VP ISE Group & Intact Animal Group & Sham Group \\
\hline Gx & 1.4748 & 1.3901 & 1.1705 & 2.8823 \\
Gy & 1.4823 & 1.3866 & 1.1461 & 2.9118 \\
CR & 1.7657 & 1.8874 & 2.5724 & 0.4485 \\
\hline
\end{tabular}

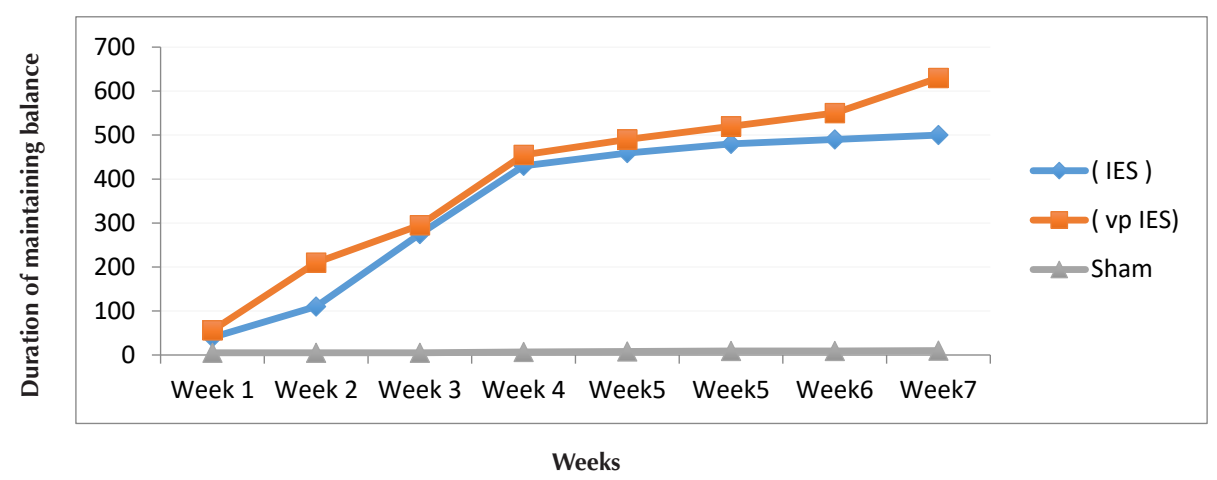

Figure 3. Evaluation of improvement in Motor Performance Using Balance Measurements.

lower limbs have paralyzed, it was shown that continuous electrical stimulation which affected their spinal cord in an area below the injured area, has contributed to modify the motor commands and it has led to motor recovery. After a period of movement training which led to maturation in the nervous system, so that motor commands can generate without the need for intervention. In this study, animals with partial paralysis of spinal cord used for investigation. Therefore, the brain and its path to the spinal cord has not discarded completely, but it has been attempted to guide the brain to go to the point where it can correct its motor comments by stimulating sensory nerves and enhancing sensory information. Meanwhile, the changes are also seen gradually in the variations of the CPG rhythm. Therefore, varying intensity of intervention can help the brain to perform its modifications gradually conforming to the situation of the neural system. Accordingly, a group of spinalized rats received the variable pattern of stimulation, in a manner that the intensity of the received intra-spinal electrical stimulations was increased in each exercise session relative to the last exercise session. In the other side, balance control is an organized activity, and various factors are affecting it. Moreover, it is one of the most important Kinematic and synergistic factors of muscle and joints during gait. If the muscles and joints of both legs of animal can coordinate when moving with the same pattern, the stability and balance can observe during gait. Therefore the aim of the present study was the recovery of the animal's gait with the appropriate balance so that the animal can move like a healthy animal. So, the improvement of the kinematic synergy should be evident during the training of animals. This improvement in kinematic synergy is noticeable by enhancing the quality of animal balance during stepping. In the current study, kinematic synergy improvement has achieved by doing the process of training on the treadmill, along with receiving an electrical stimulus. Based on an examination of the behavior of the animal in order to test the balance on a Rota-Rod Rat machine, it has seen that the animal was able to maintain a longer time on the rotating Rod after passing training sessions. This is not possible until there is synchronization of movement or kinematic synergy between joints. In fact, in order for the animal to be able to move on the surface, it is necessary to adapt its motion to different surface conditions and to ensure its sustainability by the proper and harmonious movement of the joints and the muscles of both legs. The same issue observed in the nonlinear analysis of the movement of the ankle joint of the animal. The movement dynamics of the healthy rats and the spinalized rats compared through the analysis of the constructed phase plan related to the ankle joint movement. The two features of the center of mass and the correlation sum investigated by nonlinear analysis of the phase plane. The center of mass represents the position of the points in the phase plane. The correlations sum also determines how many pairs of points among all pairs of possible points exist that the distance between them is less than a certain scale, such as $\varepsilon$. Therefore, the correlation sum represents how the distribution of points in the phase space is. Both aforementioned geometric features contain information concerning how the point located and distributed in the gait-related phase plane. This can delineate a meaningful image concerning the system dynamics. Overall, it can claim that motion recovery observed in both animal groups. Observing the motion recovery in IES group conform to the previously reported studies. ${ }^{20-22}$ However, our specific findings are related to the quality of motion recovery in VIES group. 
Because the effectiveness of such a stimulation profile has not addressed in the previous eminent studies. ${ }^{20-22}$ According to the observations, the lower the mass center values are, the less the spatial distribution of the points in the phase space is, and the more concentrated the points are. Since the value of the center of mass related to the VPIES group is less than that is related to the IES group, and it is closer to the healthy animal group. It can be stated that the gait pattern of the VPIES group is more similar to the healthy animal pattern. Also, it has observed that the data expansion along the vertical axis in the phase plane related to the VPIES group was lower than the IES group. This also emphasizes that the movement pattern of VPIES group pattern was closer to the healthy animal group in terms of not only the range of motion but also the gait dynamics.

The next most important step in the present study is to conduct human experiments. Therefore, implants producing electrical pulses in the lower part of the injured spinal cord can use in order to stimulate the nerves of the spinal cord in humans. Due to promising results in animal experiments which have obtained of the current study, it has hoped that implantation leads to recovery of the patient with regenerating the nerves in a region with spinal cord injury by using epidural electric stimulation as variable stimulation protocol.

\section{Conclusion}

Due to examining the results, it can be deduced that performing seven-week gait training on the treadmill with weight support for animals suffering from partial spinal cord injury as well as use of intra-spinal electrical stimulation techniques were able to improve the balance, muscular coordination and led to motor recovery in animals after induction of spinal cord injury. However, applying the variable intraspinal electrical stimulation along with the gait training on the treadmill, which means the variable intensity of the intervention, can give rise to better motor coordination and balance preservation after receiving the intervention. Hence, what was done in the present paper is the first step in designing a novel method and developing a new strategy in motor rehabilitation.

\section{Conflict of Interest Disclosures}

The authors declare that they have no conflict of interests.

\section{Ethical Statement}

All procedures on animals were reviewed by a local animal care committee to ensure that the procedures are appropriate.

\section{References}

1. Thuret S, Moon LD, Gage FH. Therapeutic interventions after spinal cord injury. Nat Rev Neurosci. 2006;7(8):628-43. doi: 10.1038/nrn1955.

2. Fitch MT, Silver J. CNS injury, glial scars, and inflammation: Inhibitory extracellular matrices and regeneration failure. Exp Neurol. 2008;209(2):294-301. doi: 10.1016/j. expneurol.2007.05.014.
3. Capogrosso M, Milekovic T, Borton D, Wagner F, Moraud EM, Mignardot JB, et al. A brain-spine interface alleviating gait deficits after spinal cord injury in primates. Nature. 2016;539(7628):284-8. doi: 10.1038/nature20118.

4. Franz $M$, Richner $L$, Wirz $M$, von Reumont $A$, Bergner $U$, Herzog T, et al. Physical therapy is targeted and adjusted over time for the rehabilitation of locomotor function in acute spinal cord injury interventions in physical and sports therapy. Spinal Cord. 2018;56(2):158-67. doi: 10.1038/s41393-0170007-5.

5. Myckatyn TM, Mackinnon SE, McDonald JW. Stem cell transplantation and other novel techniques for promoting recovery from spinal cord injury. Transpl Immunol. 2004;12(34):343-58. doi: 10.1016/j.trim.2003.12.017.

6. Rossignol S, Frigon A. Recovery of locomotion after spinal cord injury: some facts and mechanisms. Annu Rev Neurosci. 2011;34:413-40. doi: 10.1146/annurevneuro-061010-113746.

7. Ichiyama RM, Gerasimenko YP, Zhong H, Roy RR, Edgerton VR. Hindlimb stepping movements in complete spinal rats induced by epidural spinal cord stimulation. Neurosci Lett. 2005;383(3):339-44. doi: 10.1016/j.neulet.2005.04.049.

8. Harvey LA. Physiotherapy rehabilitation for people with spinal cord injuries. J Physiother. 2016;62(1):4-11. doi: 10.1016/j. jphys.2015.11.004.

9. Gerasimenko YP, Avelev VD, Nikitin OA, Lavrov IA. Initiation of locomotor activity in spinal cats by epidural stimulation of the spinal cord. Neurosci Behav Physiol. 2003;33(3):247-54.

10. Granat MH, Ferguson AC, Andrews BJ, Delargy M. The role of functional electrical stimulation in the rehabilitation of patients with incomplete spinal cord injury--observed benefits during gait studies. Paraplegia. 1993;31(4):207-15. doi: 10.1038/sc.1993.39.

11. Zhu Y, Uezono N, Yasui T, Nakashima K. Neural stem cell therapy aiming at better functional recovery after spinal cord injury. Dev Dyn. 2018;247(1):75-84. doi: 10.1002/ dvdy.24558.

12. Sivaramakrishnan A, Solomon JM, Manikandan N. Comparison of transcutaneous electrical nerve stimulation (TENS) and functional electrical stimulation (FES) for spasticity in spinal cord injury - A pilot randomized crossover trial. J Spinal Cord Med. 2018;41(4):397-406. doi: 10.1080/10790268.2017.1390930.

13. Raineteau O, Schwab ME. Plasticity of motor systems after incomplete spinal cord injury. Nat Rev Neurosci. 2001;2(4):263-73. doi: 10.1038/35067570.

14. Steuer I, Guertin PA. Central pattern generators in the brainstem and spinal cord: an overview of basic principles, similarities and differences. Rev Neurosci. 2019;30(2):10764. doi: 10.1515/revneuro-2017-0102.

15. Abbinanti MD, Zhong G, Harris-Warrick RM. Postnatal emergence of serotonin-induced plateau potentials in commissural interneurons of the mouse spinal cord. J Neurophysiol. 2012;108(8):2191-202. doi: 10.1152/ jn.00336.2012.

16. Acevedo J, Santana-Almansa A, Matos-Vergara N, MarreroCordero LR, Cabezas-Bou E, Diaz-Rios M. Caffeine stimulates locomotor activity in the mammalian spinal cord via adenosine A1 receptor-dopamine D1 receptor interaction and PKA-dependent mechanisms. Neuropharmacology. 2016;101:490-505. doi: 10.1016/j.neuropharm.2015.10.020.

17. Rossignol S, Dubuc R, Gossard JP. Dynamic sensorimotor interactions in locomotion. Physiol Rev. 2006;86(1):89-154. doi: 10.1152/physrev.00028.2005.

18. Seo K, Chung S-J, Slotine J-JE. CPG-based control of a turtlelike underwater vehicle. Auton Robots. 2010;28(3):247-69. 
doi: 10.1007/s10514-009-9169-0.

19. Rybak IA, Shevtsova NA, Lafreniere-Roula M, McCrea DA. Modelling spinal circuitry involved in locomotor pattern generation: insights from deletions during fictive locomotion. J Physiol. 2006;577(Pt 2):617-39. doi: 10.1113/ jphysiol.2006.118703.

20. Boyce VS, Tumolo M, Fischer I, Murray M, Lemay MA. Neurotrophic factors promote and enhance locomotor recovery in untrained spinalized cats. J Neurophysiol.
2007;98(4):1988-96. doi: 10.1152/jn.00391.2007.

21. Steuer I, Guertin PA. Central pattern generators in the brainstem and spinal cord: an overview of basic principles, similarities and differences. Rev Neurosci. 2019;30(2):10764. doi: 10.1515/revneuro-2017-0102.

22. Deng K, Szczecinski NS, Arnold D, Andrada E, Fischer MS, Quinn RD, et al. Neuromechanical Model of Rat Hindlimb Walking with Two-Layer CPGs. Biomimetics (Basel). 2019;4(1). doi: 10.3390/biomimetics4010021. 